\title{
Effects of strain rate on the cohesive properties and fracture process of a pressure sensitive adhesive
}

\author{
Ulf Stigh, Anders Biel ${ }^{*}$ \\ University of Skövde, Skövde, Sweden
}

\begin{abstract}
A B S T R A C T
Pressure sensitive adhesives provide beneficial properties for bi-material bonding and we here focus on influences of the loading rate. The cohesive law for a tape is measured using the $J$-integral method. In-situ studies of the fracture process are made. The fracture energy and cohesive law levels off below an engineering strain rate of about $2 \mathrm{~s}^{-1}$. All the cohesive laws include two peak stresses, the first is associated with nucleation of cavities and occurs at a stress level comparable to the critical stress for cavitation in rubber. The second peak stress occurs just before final fracture when walls formed between the cavities break down. Slower rates give more time for cavities to nucleate. A similarity of the effects of loading rate and ageing is also identified.
\end{abstract}

\section{Introduction}

One of the focuses of the technology road map towards lightweight design is the ability to use optimal materials. This does often require utilizing and joining diverse materials with differences in the coefficients of thermal expansion. By this, the joints may suffer high stresses and distortions even at modest changes in the ambient temperature. In Stigh et al. [1] we argue that pressure sensitive adhesive (PSA) have desirable properties that limit the thermal loads and the distortions in bi-material joints while retaining competitive toughness. This is due to the very small stiffness and large ductility of the PSA; a PSA can withstand engineering strain of the order of $10^{1}$ with a small-strain Young's modulus and strength of the order of $1 \mathrm{MPa}$ while the fracture energy is of the order of $10^{3} \mathrm{~N} / \mathrm{m}$, cf. e.g. Hayashida et al. [2] and Biel and Stigh [3]. Thus, the fracture energy is of the same order of magnitude as epoxy adhesives though the stress and stiffness are three orders of magnitude smaller. The smaller stiffness gives smaller thermal stresses in bi-material joints. It is thus desirable to improve our understanding and knowledge of the fracture properties of PSA in order to take full advantage of these adhesives in design.

In Biel and Stigh [3], properties for a cohesive layer model of a PSA are reported. A cohesive law, relating the stress to the elongation of the PSA in mode I loading shows two peaks stresses. The first peak occurs at small deformation; this is associated with nucleation of cavities in the PSA. The second peak stress occurs just before fracture. This is associated with extensive stretching of the PSA. The first peak stress shows reasonable agreement with the nucleation stress for unlimited cavity growth in rubber predicted in a theory by Gent and Lindley [4] and generalized by Ball [5]. The similarities between the present PSA and rubber are the extreme flexibility, an almost isochoric deformation, and a similar stress vs. extension relationship in macroscopic testing. The second peak stress is in reasonable agreement with results from macroscopic tests of the same PSA reported by Pharr et al. [6]. The occurrence of two peak stresses is not shown for other adhesives and appears to be a unique feature of PSA. However, the PSA is not fully incompressible and shows viscos properties. Viscoelastic properties of polymers and viscoelastic material models have been studied extensively cf. e.g. Ferry [7] and Christensen [8] for relevant overviews.

In the present paper, we extend the study in Biel and Stigh [3] to include a study of the influence of strain rate. As in that paper,

\footnotetext{
* Corresponding author.

E-mail addresses: ulf.stigh@his.se (U. Stigh), anders.biel@his.se (A. Biel).
} 
we utilize the Double Cantilever Beam (DCB) specimen with transparent substrates facilitating the possibility to directly observe the micromechanisms of the fracture process. Compared to the butt joint test configuration, the DCB-specimen is more stable. Thus, providing mechanical stability during the complete softening of the PSA.

The paper is organized with an introduction to the cohesive layer model used to model the tape and the experimental method used to measure the associated cohesive law. After this, the material and the experimental set-up are described. Special emphasise is given to the nucleation, growth and coalesce of cavities during the loading. These are studied in-situ during the experiments. The experimental results are then presented and evaluated. The paper ends with some conclusions after a section with discussion and outlook.

\section{Theoretical background}

Most lightweight structures are build-up from bars, beams and shells. These are analysed based on simplifying assumptions on the state of deformation. Most notable are the well-known assumptions identified with the names Euler and Bernoulli (beam theory), Love and Kirchhoff (shell theory), and Timoshenko and Mindlin (shear-corrected beam and shell theories), cf. e.g. Fung [9]. These theories provide beam and shell models that yield accurate results for many applications. With the cohesive layer model, a similar level of approximation is found for layers used to bond the bars, beams or shells. With the model, the deformation of the layer is given by the separation of adjacent points on the interfaces between the substrates and the layer. In this section, the necessary background is given to the model and the method used to evaluate the experiments. The section starts with an introduction of the notation used and approximations through an analysis of the stress, strain and energy state in a thin flexible layer between stiffer substrates. This is followed by an introduction to the cohesive layer model used to represent the properties of the layer. Making limited assumptions on the properties of the layer, it is shown that the path-independent property of Rice's $J$-integral can be utilized in experiments to directly measure the cohesive law of the model. This section ends with an application of the derived relations to the present experimental set-up.

In a joint, a layer, i.e. the adhesive, joins two substrates as visualized in a general principle sketch in Fig. 2.1. Loading of the layer is transmitted through the interfaces between the layer and the substrates as normal, $\sigma$, and shear, $\tau$, stresses from loads acting on the substrates. Work conjugated deformation measures are $w$ and $v$. The thickness of the layer is assumed to be small enough to yield the same $\sigma$ and $\tau$ on both the upper and lower interface for the same $x$ - and $z$-coordinate. Consider now a layer in the $x$ - $z$-plane with its major extension in the $x$-direction. The layer thickness $h$ in the $y$-direction is much smaller than the extensions in the $x$ - and $z$ directions. Strains, stresses and subsequently cavities develop in the layer when it is loaded. The conventional linearized strain components are denoted $\varepsilon_{x}, \varepsilon_{y}, \varepsilon_{z}, \gamma_{x y}, \gamma_{y z}$, and $\gamma_{x z}$, cf. e.g. Timoshenko [10]. Due to the constraints exerted by the much stiffer substrates, the in-plane strain components $\varepsilon_{\mathrm{x}}, \varepsilon_{\mathrm{z}}$, and $\gamma_{\mathrm{xz}}$ in the layer are very much smaller than the out-of-plane strain components $\varepsilon_{\mathrm{y}}$ and $\gamma_{\mathrm{xy}}$. At fracture, the out-of-plane engineering strain components can be of the order of $10^{0}$ for adhesives and $10^{1}$ for PSA (cf. e.g. [11] for epoxy and [1] for PSA). On the other hand, the substrates suffer much smaller strains and for the present experiments the inplane strain components are less than about $10^{-3}$ in the substrates. By continuity, the in-plane strain components in the layer are of the same order of magnitude. The out-of-plane strain components of the engineering strain in the substrates are at least one order of magnitude smaller considering their beam like geometry and loading, cf. e.g. Timoshenko [10]. For the present purpose, we will also assume that the sixth strain component $\gamma_{\mathrm{yz}}$ is very small since test specimens often are loaded in the $x$ - $y$-plane. Based on these observations, the strain state in the layer is simplified by assuming $\varepsilon_{x}=\varepsilon_{z}=\gamma_{x z}=\gamma_{y z}=0$. For small strains and a homogeneous layer, the separation of two adjacent points on the interfaces of the layer and the substrates is given by

$$
w=\int_{h} \varepsilon_{y} \mathrm{~d} y v=\int_{h} \gamma_{x y} \mathrm{~d} y
$$

These variables are used as the basic deformation measures for the layer; $w$ being the peel deformation and $v$ the shear deformation. It is noted that in a general three-dimensional setting, the shear deformation needs to be split in two non-parallel components. It is convenient to keep these as deformation measures when reporting the mechanical properties of a layer since the toughness of an adhesive layer depends on the thickness of the layer, cf. e.g. Kinloch [12]. The alternative, to present the data per unit thickness, i.e. using strain instead of deformation as the measure of deformation, may lead to the erroneous assumption that the data scales linearly with the layer thickness. This is obviously in error since the fracture energy is experimentally observed to scale in a much more complicated way with the thickness of the layer.

Denoting the work-conjugated stress measures $\sigma$ and $\tau$ yields the work performed on the layer per unit area $W$ in the $x$ - $z$-plane

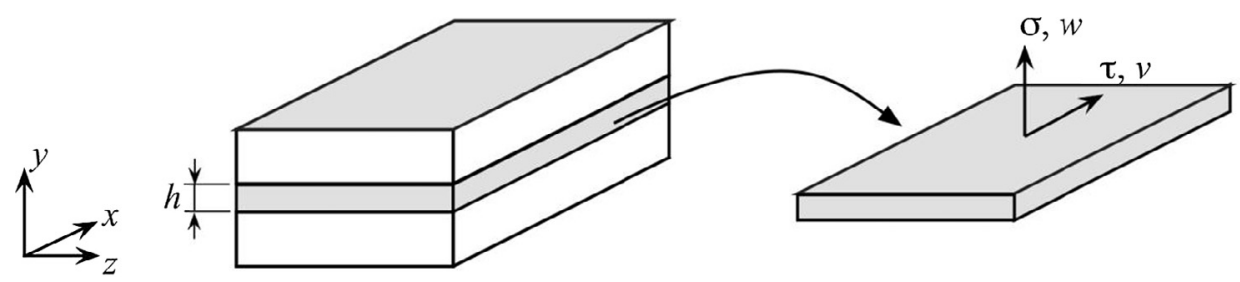

Fig. 2.1. Normal and shear deformation and stress of a layer with thickness $h$. 


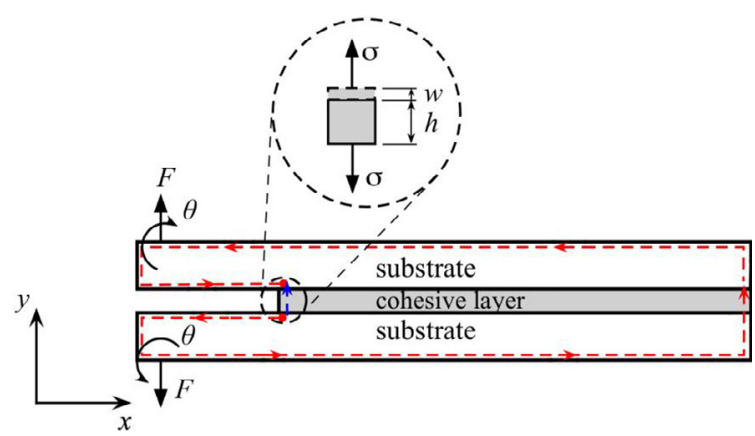

Fig. 2.2. Idealized DCB-specimen. The red integration path is used to derive the relation between external loads and $J$, i.e. $J=2 F \theta / b$. The blue integration path is used to derive the relation between the cohesive law and $J$ in Eq. (2.5). (For interpretation of the references to color in this figure legend, the reader is referred to the web version of this article.)

$$
W(w, v)=\int_{0}^{w} \sigma \mathrm{d} w+\int_{0}^{v} \tau \mathrm{d} v .
$$

During a loading history with monotonically increasing deformation $(w, v), \sigma$ and $\tau$ will first increase, corresponding to a linear elastic response of the layer, and subsequently decrease, corresponding to the development of cavities in the layer. Finally, when the cavities coalesce, corresponding to the formation of a macroscopic crack, $\sigma=\tau=0$. Thus, the formation and growth of cavities are implicitly taken into account by a gradual decrease in traction as the deformation increases.

Consider now the start of the layer in a test specimen - denoted the crack tip, cf. e.g. Fig. 2.2. Due to the symmetry of the specimen, $v \equiv 0$ during loading and $W$ reduces to be a function of $w$ only. Eq. (2.2) thus shows that $W(w)$ is a potential for the cohesive stress $\sigma$, i.e.

$$
\sigma(w, v)=\frac{\partial W}{\partial w}
$$

The present experimental procedure focuses on measuring $W(w)$. The method presented below shows how this energy can be measured experimentally.

The present experimental procedure is developed based on the path-independent property of Rice's $J$-integral.

$$
J=\int_{S}\left(U \mathrm{~d} y-T_{i} u_{i, x} \mathrm{~d} S\right)
$$

where $U$ is the strain energy density; $T_{i}$ and $u_{i}$, are the $i$-components of the traction and displacement vectors, respectively, where $i$ takes the values $x$ and $y$, a comma indicates partial differentiation, $S$ is the integration path, and dy is the vertical component of d $S$. It is straightforward to show that $J=0$ for any integration path encircling a part of an elastic body provided certain conditions are met, cf. Rice [13]. The conditions can be summarized by two conditions. Firstly, the existence of the function $U$. This function is not allowed to be explicitly dependent on the $x$-coordinate. That is, the material is allowed to be non-linear hyperelastic and it is also allowed to have differences in the material properties in the vertical direction but not in the horizontal direction. Secondly, $S$ is not allowed to encircle any identifiable features that would change the energy of the body if they were moved in the horizontal direction. Such features can be boundaries and applied loads.

Consider now the closed integration path formed by combining the red and blue integration paths in Fig. 2.2. Inside this path there are no features that would change the energy of the body if they were moved horizontally. Furthermore, the material properties are homogeneous in both the layer and the substrates in the $x$-direction. Thus, for this path $J=0$. Consider now the contribution from the red and blue integration paths separately; denote these $J_{\mathrm{F}}$ and $J_{\mathrm{A}}$ respectively and note that $J=J_{\mathrm{F}}-J_{\mathrm{A}}=0$, where the minus sign originates from the definition of the positive direction chosen for the blue integration path, cf. Fig. 2.2. Assuming the specimen to be long enough, the only features changing the energy are the forces $F$ and the start of the layer. That is, $U$ is assumed negligibly small at the left and right boundaries of the specimen, cf. Fig. 2.2. An evaluation of Eq. (2.4) for the red integration path gives $J_{\mathrm{F}}=2 F \theta / b$, cf. Paris and Paris [14] and Biel and Stigh [15], where $b$ is the width of the specimen in the out-of-plane direction. For the blue integration path, Eq. (2.4) gives

$$
J_{\mathrm{A}}=\int_{h} U \mathrm{~d} y=\int_{h}\left(\int_{0}^{\epsilon_{y}} \sigma_{y} \mathrm{~d} \epsilon_{y}\right) \mathrm{d} y=\int_{0}^{\epsilon_{y}}\left(\int_{h} \sigma_{y} \mathrm{~d}_{y}\right) \mathrm{d} \varepsilon_{y}=\int_{0}^{\varepsilon_{y}} \sigma h \mathrm{~d} \varepsilon_{y}=\int_{0}^{w_{0}} \sigma \mathrm{d} w=W .
$$

where the first equality follows from the traction free condition at the start of the blunted layer. The second equality follows from the definition of the strain energy density and by setting the other strain components equal to zero as discussed above. After switching the order of integration in the third equality, the fourth equality shows that the normal stress, $\sigma$ is the averages of the local stresses $\sigma_{y}$ taken over the thickness $h$ of the layer. Finally, $W$ according to Eq. (2.2), is identified after changing integrating variables from the strains to the separation $w_{0}$ at the crack tip using Eq. (2.1). Using the results above, we arrive at

$$
\sigma\left(w_{0}\right)=\frac{2}{b} \frac{\partial(F \theta)}{\partial w_{0}}
$$


The index 0 indicates that $w$ is measured at the start of the layer. Now, in the derivation of Eq. (2.5), we have assumed that the same law $\sigma(w)$ is valid for the complete layer. This means that the index 0 can be dropped after the differentiation in Eq. (2.6). The same relation holds for all points, not just for the start of the layer. It is noted that Olsson and Stigh [16] originally derived this result using a different method.

\section{Experiments}

An acrylic, closed cell transparent PSA (3M VHB-4905F) with thickness $h=0.5 \mathrm{~mm}$ is used to bond transparent PMMA substrates. Nominal elastic properties ${ }^{1}$ of the materials are given by Young's modulus $3 \mathrm{GPa}$ (PMMA) and $0.5 \mathrm{MPa}$ (PSA). Poisson's ratio is 0.35 (PMMA) and 0.49 (PSA), respectively. Thus, the PSA is nearly incompressible. Note that these data do not explicitly enter the equations used to evaluate the experiments, cf. Eq. (2.6). The PSA has been studied extensively by e.g. Plante and Dubowsky [17], Zhao and Suo [18] and Pharr et al. [6]. The nominal dimensions of the DCB-specimens are $H=5.7 \mathrm{~mm}, a=60 \mathrm{~mm}, l=190 \mathrm{~mm}$, $b=B=19 \mathrm{~mm}$, cf. Fig. 3.1. The length $l-a=130 \mathrm{~mm}$ is large enough to secure that the right end of the specimen is virtually stress free, thus Eq. (2.6) can be used. PSA from the same roll of tape is used in the present study as was used in the study by Biel and Stigh [3]. In that study, only one loading rate was studied, i.e. $\dot{\Delta}=24 \mu \mathrm{m} / \mathrm{s}$. Those results are reproduced below together with the results from the present study.

The specimens are joined at least $72 \mathrm{~h}$ before the experiments to conform to the recommendations of the manufacturer. During this time, a contact pressure is applied by rubber bands. ${ }^{2}$ All experiments are performed at room temperature using a custom-built electro-mechanical test machine (Swetest 100-168), cf. Fig. 3.2. The machine is designed for testing of DCB-specimens and operates by symmetrically separating the two crossheads at a prescribed velocity $\dot{\Delta}$. The DCB-specimens are attached using ball bearings to minimize unintentional application of bending moments due to friction. LVDTs are used to measure the peel deformation $w_{0}$ at the start of the layer. The build-in load cell is used to measure the applied force $F$ and a shaft encoder is used to measure the rotation $\theta$.

The transparent material allows for in-situ observations of the fracture mechanisms inside the PSA. A time-synchronized camera (Nikon D300) with a macro lens is used to take images through the thickness of the tape (viewing in the $y$-direction). To improve the contrast, a black paper is used on the opposite side of the specimen and the edges of the specimen are illuminated. The illumination gives reflections of the edges of voids formed during the loading. Thus, the voids are clearly identified, cf. Biel and Stigh [3] where the same set-up is utilized.

To measure the cohesive law for the tape using Eq. (2.6), the crossheads are separated with a constant rate $\dot{\Delta}$. During an experiment, the reaction force $F$, the rotation of the loading points $\theta$ and the elongation $w_{0}$ of the tape are measured with a suitably chosen frequency. About $10^{4}$ data points are recorded for each experiment; images are taken less frequently and about $10^{2}$ images are recorded during an experiment. In Biel and Stigh [3] butt joint experiments are performed that show that the effects of the viscoelastic properties of the tape only marginally disturbs the evaluation using Eq. (2.6).

Four sets of experiments are performed to study the rate dependence. Results in Biel and Stigh [3] indicate an almost linear relation between the elongation $w_{0}$ and $\Delta$ during the most part of an experiment. Thus, we may expect $\dot{w}_{0} \sim \dot{\Delta}$ and the rates $\dot{\Delta}=8.0$, 24,100 and $530 \mu \mathrm{m} / \mathrm{s}$ are chosen to be about evenly separated on a logarithmic scale.

\section{Experimental results}

Fig. 4.1 shows the force $F$ vs. load point displacement $\Delta$ for all experiments. Some limited scatter in experimental data is visible for each group of experiments. For smaller loads, $F$ increases almost linearly with $\Delta$ corresponding to a linear elastic behaviour of the material. The properties of the substrate PMMA dominate the behaviour at this phase of an experiment and the curves show only small effects of the loading rate. At larger load, the inelasticity of the PSA is detected and the curves change to a new virtually constant slope. This second, phase ends when a crack forms and starts to propagate. After that, the force decreases. The maximum forces are about $46,50,50$, and $68 \mathrm{~N}$, respectively for the larger to smaller loading rates. The specimen to specimen variation is smaller than about $\pm 5 \%$.

The smaller the loading rate, the smaller the load, as expected for a viscoelastic material. Somewhat unexpectedly, it is observed that the differences between the smaller load rates are small.

Fig. 4.2 shows the average engineering strain $\bar{\epsilon}_{y} \equiv w / h$ of the PSA vs. time. Since, $\Delta$ increases linearly with time, the graph is a scaled version of a $w$ vs. $\Delta$ graph. After a parabolic start, the curves show a virtual linear relation between $w$ and $\Delta$. The linear part corresponds to constant strain rates $\dot{w}_{0} / h$ : 0.0030 (red), 0.011 (black), 0.037 (green) and 0.18 (blue) s ${ }^{-1}$, respectively, for increasing loading rates.

The evolution of $J=2 F \theta / b$ with the elongation $w$ is given in Fig. 4.3. The curves start with a parabolically increasing $J$, for very small $w$, cf. Fig. 4.3a. Recollecting that $J=W$, i.e. the potential for the cohesive stress (Eq. (2.5)), it is understood that this part of the curves corresponds to a linear elastic response of the PSA. After this part, the curves seemingly adapt to a new almost parabolic part that ends at a virtually constant part. These latter parts of the curves correspond to the propagation of a crack front at an almost

\footnotetext{
${ }^{1}$ Poisson's ratio for PMMA is given by Mott et al. [19], Young's modulus is taken from Ashby and Jones [20]. Young's modulus and Poisson's ratio for the PSA are given in the manufacturer's data sheet.

${ }^{2}$ One of the reviewers made us aware of possible problems emerging from poor control of the pressure during the joining procedure. However, we did not identify any problems with lack of adhesion during our experiments. Fracture is always initiated well inside the tape and final fracture appears to initiate by the breaking down of walls formed between the cavities. We therefore conclude that the present procedure is adequate.
} 

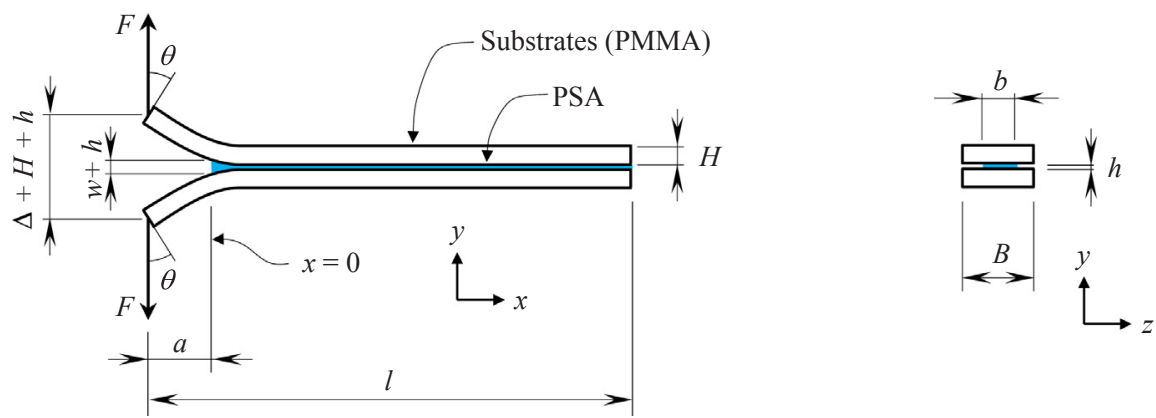

Fig. 3.1. Schematic sketch of the deformed DCB-specimen with notation and applied loads.

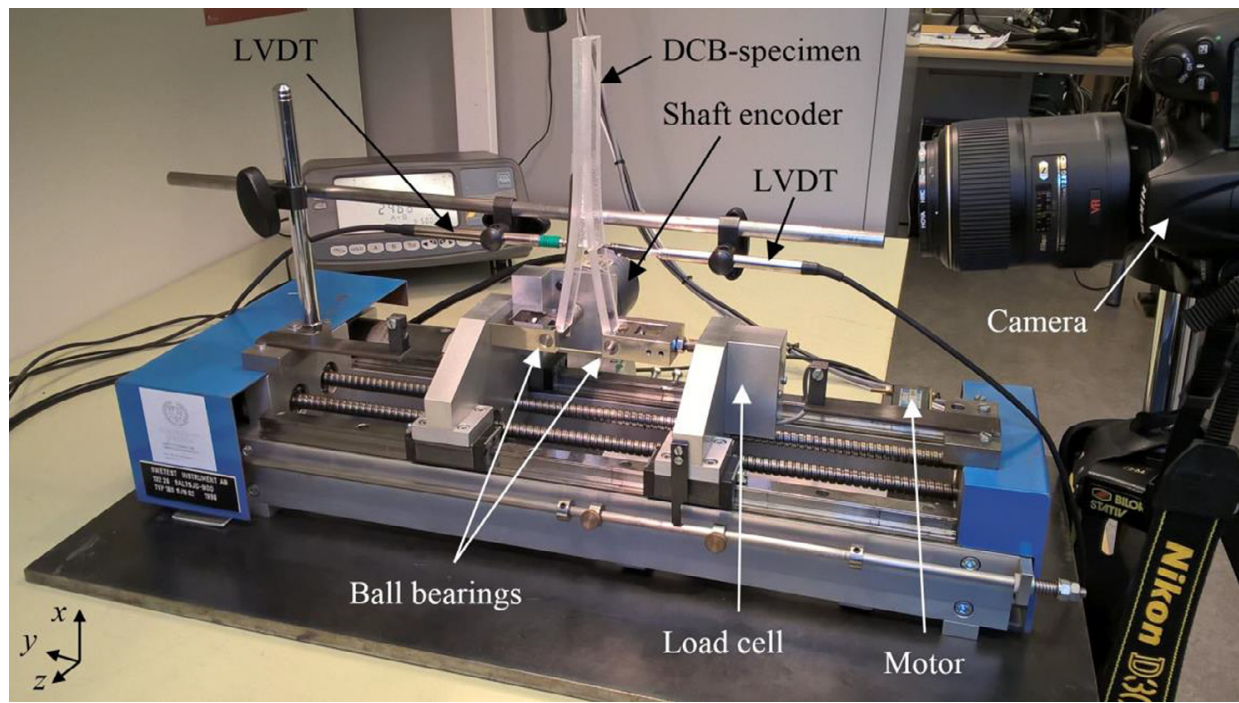

Fig. 3.2. Test machine with specimen and camera.

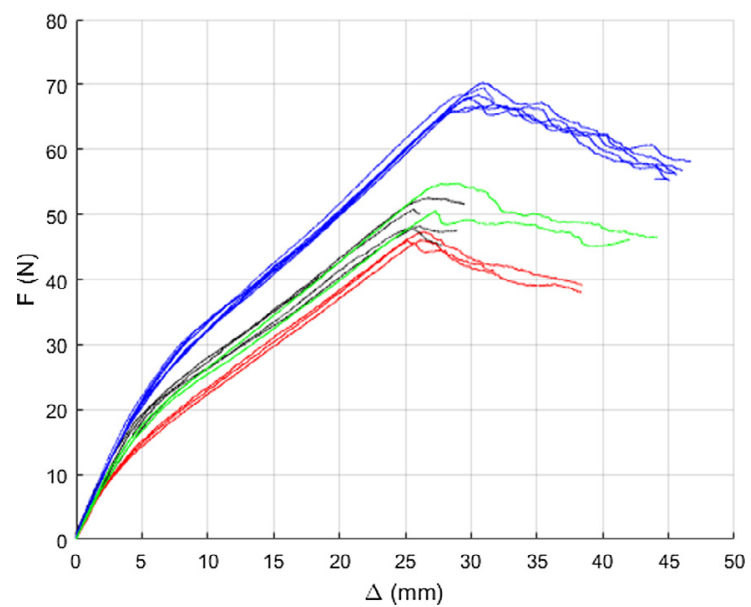

Fig. 4.1. Force vs. load point displacement for repeated experiments at four different loading rates $\dot{\Delta}=8.0$ (red), 24 (black), 100 (green) and 530 (blue) $\mu \mathrm{m} / \mathrm{s}$. (For interpretation of the references to color in this figure legend, the reader is referred to the web version of this article.)

constant fracture energy $J_{\text {Ic }}$, cf. Table 4.1. These, almost horizontal parts of the curves undulate slightly with increasing $w$. That is, with the propagation of the crack. This is attributed to minor local differences in the fracture properties of the PSA.

Differentiation of the curves in Fig. 4.3 according to Eq. (2.6) gives the cohesive stress $\sigma$ vs. elongation $w$, cf. Fig. 4.4. After a first peak stress at $\hat{\sigma}_{1}$ the stress rapidly decreases to a minimum at about one millimetre elongation, i.e. at about $200 \%$ engineering strain. Eventually, the stress starts to increase again to a second peak stress $\hat{\sigma}_{2}$. The values of $\hat{\sigma}_{1}$ and $\hat{\sigma}_{2}$ are given in Table 4.1. 


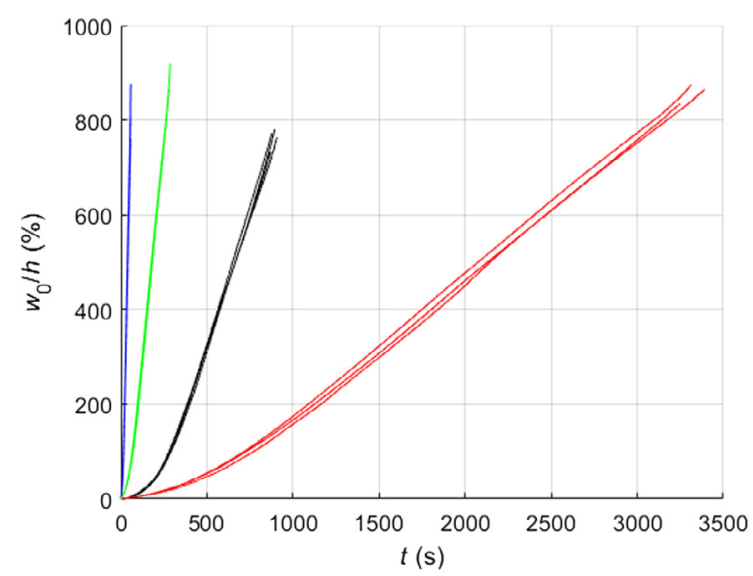

Fig. 4.2. Engineering strain vs. time for repeated experiments at four different loading rates $\dot{\Delta}=8.0$ (red), 24 (black), 100 (green) and 530 (blue) $\mu \mathrm{m} / \mathrm{s}$. (For interpretation of the references to color in this figure legend, the reader is referred to the web version of this article.)

a)

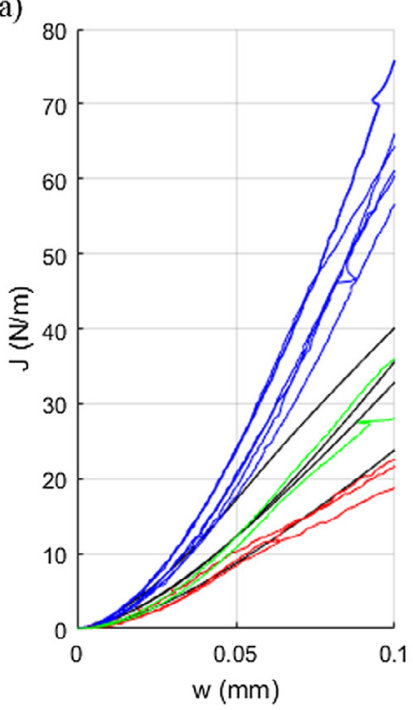

b)

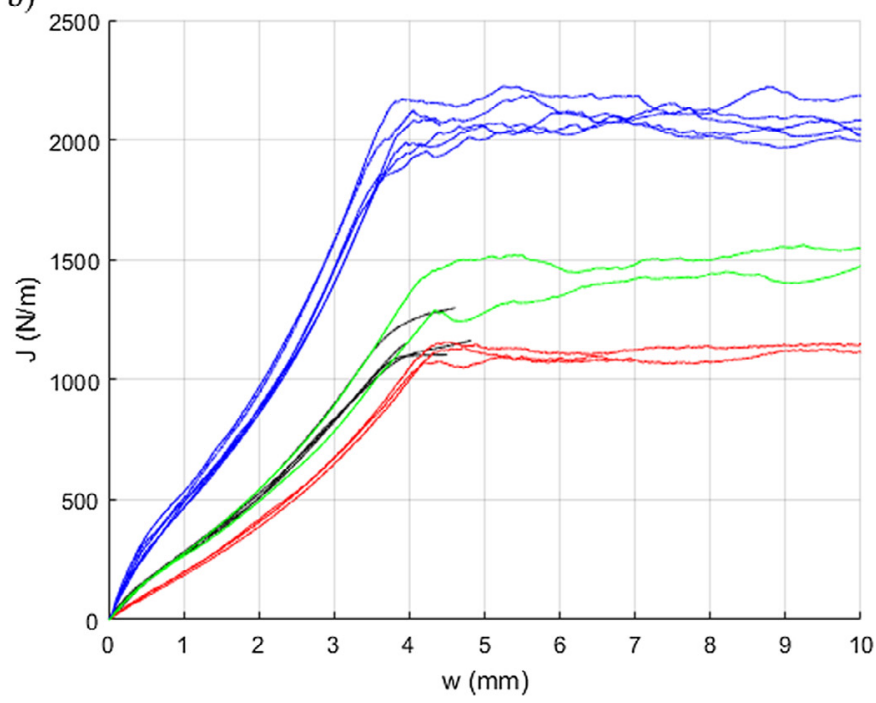

Fig. 4.3. $J$ vs. elongation of PSA for repeated experiments at four different loading rates $\dot{\Delta}=8.0$ (red), 24 (black), 100 (green) and 530 (blue) $\mu \mathrm{m} / \mathrm{s}$. a) Magnification of the first parabolic parts of the curves and b) the entire curves with crack propagation. (For interpretation of the references to color in this figure legend, the reader is referred to the web version of this article.)

Table 4.1

Mean values of evaluated data from the experiments.

\begin{tabular}{lllll}
\hline$\dot{\Delta}(\mu \mathrm{m} / \mathrm{s})$ & $\dot{w} / h\left(\mathrm{~s}^{-1}\right)$ & $J_{\text {Ic }}(\mathrm{N} / \mathrm{m})$ & $\hat{\sigma}_{1}(\mathrm{MPa})$ & $\hat{\sigma}_{2}(\mathrm{MPa})$ \\
\hline 8 & 0.0030 & 1070 & 0.21 & 0.39 \\
24 & 0.011 & 1150 & 0.42 & 0.38 \\
100 & 0.037 & 1460 & 0.70 & 0.43 \\
530 & 0.18 & 2070 & 0.70 \\
\hline
\end{tabular}

The peak stresses dependence on the engineering strain rate is shown in Fig. 4.5. The first peak stress, i.e. the circles, show a decreasing level with a decreasing strain rate. However, the second peak stress levels off for the two smallest strain rates. Note that $\sigma$ is the nominal stress. That is, for the first peak stress, reached at small deformations, the stress is virtually equal to the true stress acting on the polymer. However, for the second peak stress, large cavities have formed and the nominal stress is only a small fraction of the local true stress acting on the polymeric walls between the cavities, cf. Biel and Stigh [3].

Fig. 4.6 shows consecutive images from the fracture process at the four different loading rates and at four different elongations of the layer. The crack propagation direction ( $x$-coordinate) is downwards in all images. With the exception of the results for $24 \mu \mathrm{m} / \mathrm{s}$, the images suggest that the number of cavities increases when the loading rate decreases. The images for $24 \mu \mathrm{m} / \mathrm{s}$ are taken from the 


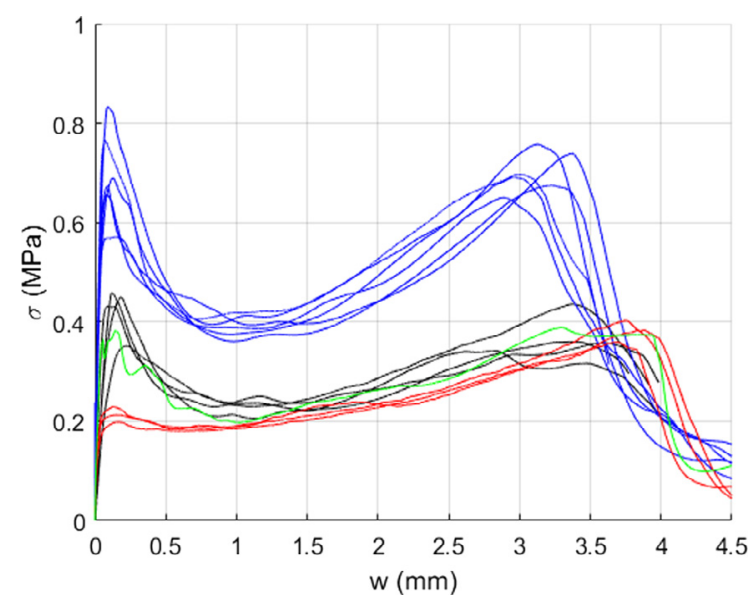

Fig. 4.4. Stress vs. deformation of PSA for repeated experiments at four different loading rates $\dot{\Delta}=8.0$ (red), 24 (black), 100 (green) and 530 (blue) $\mu \mathrm{m} / \mathrm{s}$. (For interpretation of the references to color in this figure legend, the reader is referred to the web version of this article.)

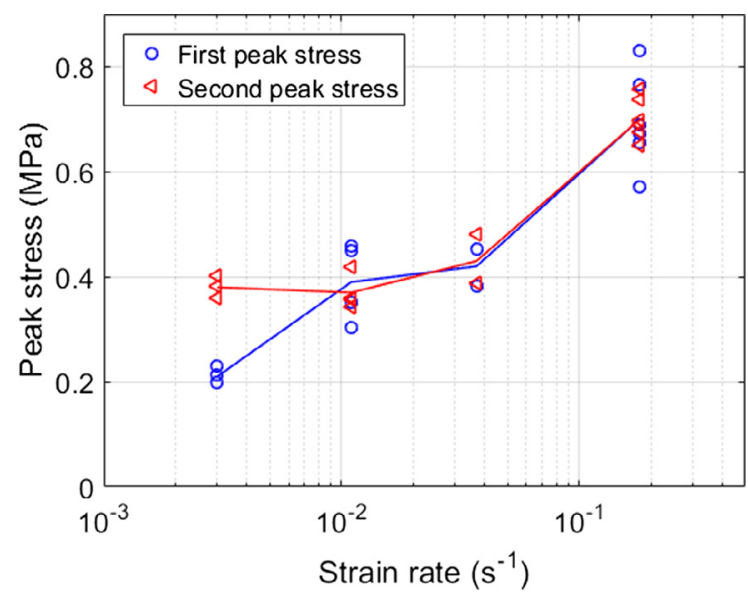

Fig. 4.5. Peak stress vs. engineering strain rate for the PSA.

earlier study [3]. In the early stage of loading, cavities nucleate; at $w_{0}=0.1 \mathrm{~mm}$ several cavities have formed close to the crack tip. All cavities nucleate within the tape. That is, no cavity nucleation is observed at the interfaces between the PMMA and the PSA. At the later stage of loading, the cavities coalesce to form a macroscopic crack, cf. the images for $w_{0}=4.0 \mathrm{~mm}$. This process is identified to be accompanied by the formation of small inlets of air in the walls between the cavities, for details cf. Biel and Stigh [3].

\section{Discussion and outlook}

The acrylic transparent PSA 3M VHB-4905F shows strain-rate dependent material behaviour. This has also been demonstrated in earlier studies, cf. e.g. Pharr et al. [6]. It has also been demonstrated for similar PSA, cf. e.g. Townsend et al. [21,22] and Hayashida et al. [2]. Biel and Stigh [3] demonstrates, by comparisons with butt joint tests, that the present experimental method provides the cohesive law although the conditions for path independence are not fully satisfied. That is, the errors that are introduced by the viscous behaviour of the PSA are of minor influence. Pharr et al. [6] report the macroscopic fracture energy in pure shear for the same PSA in the range of engineering strain rates $0.002-1.7 \mathrm{~s}^{-1}$. Their experiments are performed on a $152.4 \mathrm{~mm}$ wide (crack growth direction) and $10 \mathrm{~mm}$ long (loading direction) strips of the PSA constrained in the width direction to give a state of pure shear for an incompressible solid. Fig. 5.1 shows the fracture energy vs. strain rate for the two studies.

The results from the two studies are in agreement. However, our new results show consistently slightly smaller values of the fracture energy. It is interesting to note that similar results are obtained although the length scales are very different. In Pharr et al. [6], the length of a specimen is $152.4 \mathrm{~mm}$ while a typical cell wall in the present study is about $2 \mathrm{~mm}$ long, cf. Fig. 4.6 . Both studies indicate that the fracture energy level of at about $1 \mathrm{kN} / \mathrm{m}$ for low strain rates. This value of fracture energy is comparable to structural epoxy adhesives, cf. Carlberger et al. [23]. The epoxy adhesive DOW-Betamate XW1044-3 shows that the fracture energy is virtually linearly dependent on the logarithm of the strain rate at about $1 \mathrm{kN} / \mathrm{m}$.

For engineering use of the PSA, the long-time properties should be considered. Townsend et al. [22] report creep experiments on a 


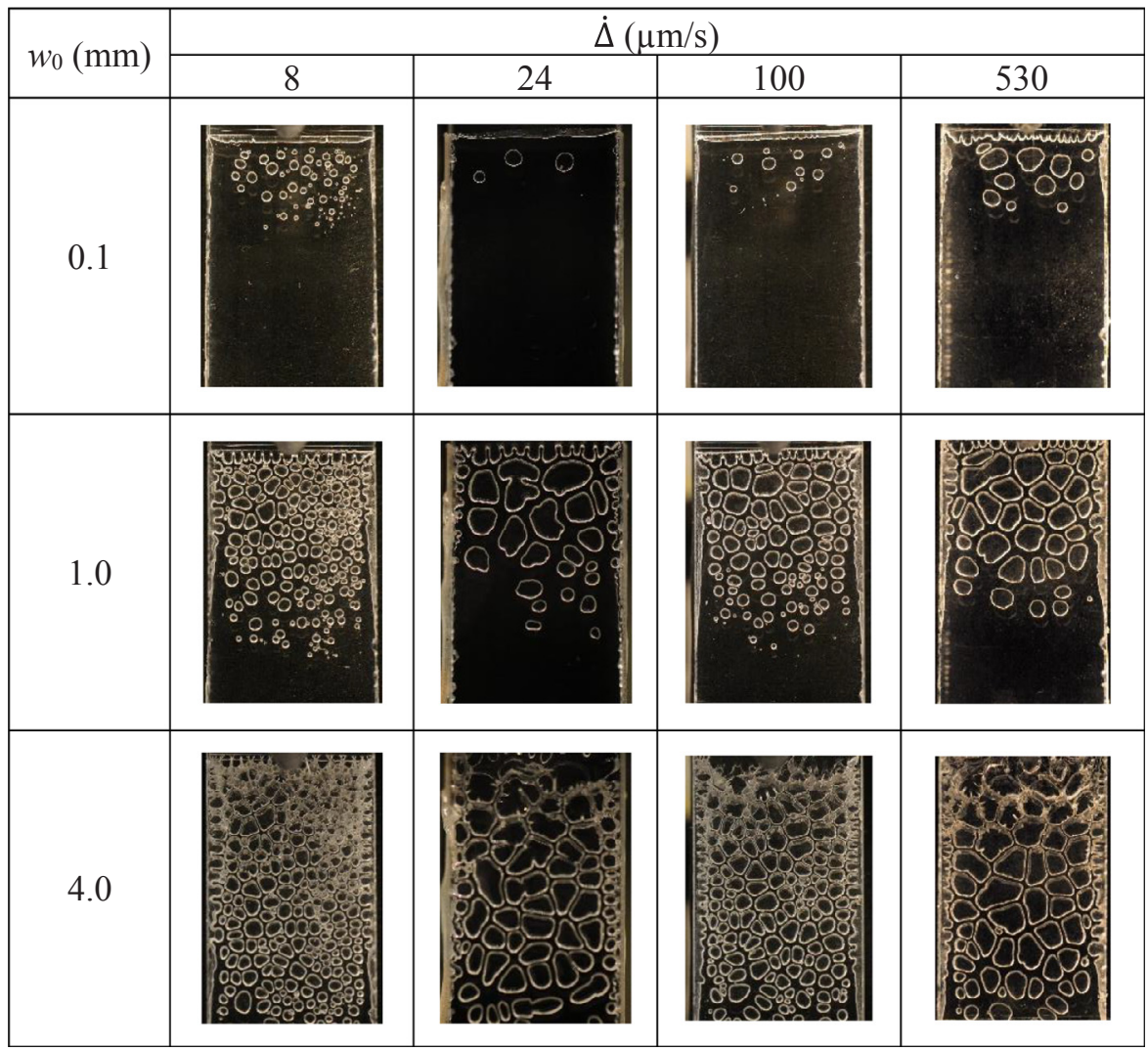

Fig. 4.6. Images at different elongation $w$ of the PSA at the different loading rates $\dot{\Delta}$.

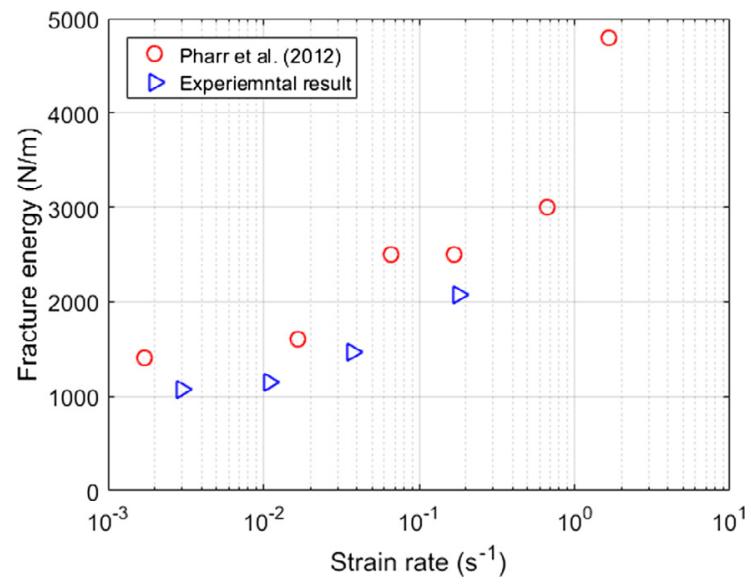

Fig. 5.1. Fracture energy vs. strain rate from Pharr et al. [6] circular red circles and the present study blue triangles. (For interpretation of the references to color in this figure legend, the reader is referred to the web version of this article.)

similar PSA (3M VHB G23F) as the one used in the present study. These show no limit stress for creep fracture. Although this is another acrylic material, more studies are advised on long time behaviour of the present PSA.

All experiments show virtually constant fracture energy for crack propagation. Thus, a steady state develops soon as the crack starts to propagate. It is sometimes thought that steady state is associated with the propagation of a stationary process zone. As illustrated by Stigh [24], this is not the case with cohesive layer modelling of the DCB-specimen loaded by applied transversal forces. Here, the process zone develops its maximum size at the moment of initiation of crack propagation. The process zone then decreases in size to a steady state size. That size equals the one for steady state cracks propagation with applied bending moments. The cohesive law and the properties of the substrates determine the steady state size of the process zone.

As illustrated in the images in Fig. 4.6, fracture of the tape is governed by nucleation, growth and coalesce of cavities. At a 
comparably small deformation, cavities nucleate within the PSA. Thus, no cavities are observed to be formed at the interfaces at this stage. Nucleation occurs at a stress level virtually coinciding with the critical stress for cavity growth in rubber elasticity, cf. Gent and Lindley [4]. The stress level is deduced from the measured cohesive law, cf. Fig. 4.4. The elasticity problem studied by Gent and Lindley [4] is the growths of a spherical cavity in a radially loaded large sphere of incompressible rubber. The sphere grows gradually as the load increases until a critical value is reached at which the growth rate becomes unbounded. Ball [5] generalized the study to include a large class of rubber elasticity models. With $E$ denoting the small strain Young's modulus of the large strain rubber elasticity model, the critical stress is given by $5 E / 6$. The first appearances of visible cavities are reasonably well correlated with this stress level. Poisson's ratio of the PSA is 0.49 , i.e. it is not fully incompressible. However, the stress state is closely approximated as a hydrostatic stress due to the constraints of the much stiffer substrates. That is, the stress for nucleation seems to be well represented by the critical stress for unbonded cavity growth rate for an isolated cavity. Now, the PSA is constrained between the PMMA substrates. This means that the growth of a spherical cavity is hindered and the cavities develop the shape of a prolonged cylinder in the $y$-direction. The expansion in the $x$-z-plane is also constrained by other cavities that grow in a similar manner, cf. Fig. 4.6 with $w_{0}=1.0 \mathrm{~mm}$. After this phase, walls form between the cavities. The walls are essentially stretched in the $y$-direction with very limited straining in its direction in the $x$-z-plane. That is, the walls are deformed similarly as in the macroscopic loading of the PSA in the study of Pharr et al. [6]. Apart from the obvious difference in dimensions between that study and the present, the cavities are here deduced to be in a state close to vacuum, cf. Biel and Stigh [3]. The macroscopic stress gradually increases as the loading of the specimen increases. It finally reaches a new maximum, cf. Fig. 4.4, after which the walls between the cavities fracture and a macroscopic crack is formed. The phenomenon shows interesting similarities with the Rayleigh-Taylor instability of the interface between two fluids of different densities that occurs when the lighter fluid is pushing the heavier fluid, cf. Rayleigh [25] and Taylor [26].

The ratio in strain rate between for the fastest and slowest strain rate in the present study is $17 / 0.3 \approx 60$. The data in Pharr et al. [6] is hard to evaluate. However, we estimate that this ratio in strain rate corresponds to about halving Young's modulus. The present results are in reasonable agreement with this deduction.

The second peak stress $\hat{\sigma}_{2}$ appears to be related to the stress at a critical stretch ${ }^{3}$ of the acrylic polymer remaining as walls between the cavities formed during the fracture process, cf. Biel and Stigh [3]. That is, using a fracture criterion for the walls between the cavities in terms of a critical stretch for the material. The present experiments indicate a slight decrease in this critical stretch as the strain rate is increasing, cf. Fig. 4.4. At about this moment in an experiment, tunnels form in the walls between the cavities and air finds its way into the cavities. This releases the relative vacuum inside the previously formed cavities. This result in a new rapid decrease of the macroscopic cohesive stress and final fracture at a critical stretch. Considering the small cohesive stresses and the relative vacuum in the cavities, the influence of the ambient air pressure should be considered for applications at other environments, cf. Biel and Stigh [3].

The cohesive stress vs. elongation relations in Fig. 4.4 show unexpectedly small influences of the strain rate for the smaller strain rates, i.e. 0.30 and $1.1 \mathrm{~s}^{-1}$. No major difference is seen even at $3.6 \mathrm{~s}^{-1}$, i.e. for smaller strain rates, the cohesive law appears to level off. This is, to some extent consistent with the result regarding the fracture energy of Pharr et al. [6].

The fracture process, as illustrated in Fig. 4.6, show very similar features at all strain rates. Biel and Stigh [3] give a detailed description for the process at the strain rate $1.1 \mathrm{~s}^{-1}$. It appears as if a smaller strain rate gives smaller cavities. This would indicate that time is influential in the process of nucleating cavities, i.e. longer time gives more nucleuses. Hayashida et al. [2] studied the strain rate dependence for another PSA and showed that that the PSA change to a brittle behaviour at the highest strain rate. Thus, no cavities nucleate at that rate. Our observation of a rate dependent nucleation process is consistent with this. Thus, at high strain rate, no time is available for the process of nucleating cavities. In the present study, the experiment not consistent with this trend is the one at $24 \mu \mathrm{m} / \mathrm{s}$. Images for this loading rate are taken from the earlier study by Biel and Stigh [3]. PSA from the same roll is used in both sets of experiments and the same procedure of specimen manufacturing is used. The only relevant differences are related to aging where the present study is performed on PSA that is almost three years older than in the earlier study. The PSA has been stored at room temperature. The time stored is larger than the 24 months recommended by the manufacturer. It is interesting to note that the macroscopic results for the older tape (new experiments) loaded at $100 \mu \mathrm{m} / \mathrm{s}$ are very similar to the results at $24 \mu \mathrm{m} / \mathrm{s}$ with a newer tape (old experiments), cf. green and black curves in Figs. 4.1, 4.3, and 4.4. That is, aging appears to have a similar effect as strain rate on the macroscopic properties of this PSA. To make the older tape react as the newer, the strain rate has to increase. The result suggests the relevance of an internal time-scale $\bar{t}=t / t_{\text {age }}$ for the present material where $t_{\text {age }}$ is the age of the material. That is, the cohesive law that the results suggest is a function of deformation, rate of deformation, and aging time $t_{\mathrm{age}}$, i.e. $\sigma\left(w, \dot{w}, t_{\mathrm{age}}\right)$. The observation above suggests that this relation should be possible to simplify as $\sigma\left(w, \frac{\mathrm{d} w}{\mathrm{~d} t}\right)$. There is obviously too little experimental evidence to claim that this is true. However, an experimental study is suggested for the future.

The rubber elasticity theory used in Biel and Stigh [3] to correlate to the nucleation stress lacks influences of time, cf. Gent and Lindley [4] and Ball [5]. Thus, a similar analysis considering viscous properties would be interesting for a comparison with the present results. These indicate that the higher stiffness associated with a higher strain rate for a viscous material can be one explanation of the transition to a brittle behaviour for pressure sensitive adhesives at high strain rates. Alternatively, Villey et al. [27] identifies large strain rheology as responsible for the rate dependence of PSA.

\footnotetext{
${ }^{3}$ Stretch is defined as deformed length divided with original length, cf. e.g. Fung [9]. A critical stretch is a limiting value at which fracture occurs.
} 


\section{Conclusions}

As shown in an earlier study [3], the cohesive law associated with mode I loading of the pressure sensitive tape 3M 4905F contains two peak stresses at several loading rates. The first peak stress is associated with the nucleation of cavities within the tape; the second by the stretching and failure of walls formed between the cavities. The strain rate dependence levels off at a strain rate below about $2 \mathrm{~s}^{-1}$. The fracture energy appears to level off at about $1 \mathrm{kN} / \mathrm{m}$. Irrespective of the loading rate, similar micromechanisms of cavity nucleation and growth are observed. However, aging changes the process of cavitation of the tape. The cavity density increases with aging.

Macroscopically, aging appears to give similar influences as decreasing the strain rate. This suggests that the macroscopic properties might be possible to represent by a master curve that scales with aging using an internal time scale related to age.

\section{Acknowledgement}

We are grateful to Dr. Roger Hagen 3M for providing the PSA to this study.

\section{References}

[1] Stigh U, Biel A, Svensson D. Cohesive zone modelling and the fracture process of structural tape. Procedia Struct Integrity 2016;2:235-44.

[2] Hayashida S, Sugaya T, Kuramoto S, Sato C, Mihara A, Onuma T. Impact strength of joints bonded with high-strength pressure-sensitive adhesive. Int J Adhes Adhes 2015;56:61-72.

[3] Biel A, Stigh U. Cohesive zone modelling of nucleation, growth and coalesce of cavities. Int J Fract 2017;204:159-74.

[4] Gent AN, Lindley PB. Internal rupture of bonded rubber cylinders in tension. Proc Roy Soc London. Series A, Math Phys Sci 1959;249(1257):195-205.

[5] Ball JM. Discontinuous equilibrium solutions and cavitation in nonlinear elasticity. Philos Trans R Soc Lond Series A, Mathe Phys Sci 1982;306(1496):557-611.

[6] Pharr M, Sun J-Y, Suo Z. Rupture of a highly stretchable acrylic dielectric elastomer. J Appl Phys 2012;111:104114.

[7] Ferry JD. Viscoelastic properties of polymers. 3rd ed. New York: Wiley; 1980.

[8] Christensen RM. Theory of viscoelasticity. 2nd ed. New York and London: Academic Press; 1982.

[9] Fung YC. Foundations of solid mechanics. Englewood Cliffs, N.J.: Prentice-Hall; 1965.

[10] Timoshenko SP. Theory of elasticity. 3rd ed. Auckland: McGraw-Hill; 1970.

[11] Andersson T, Biel A. On the effective constitutive properties of a thin adhesive layer loaded in peel. Int J Fract 2006;141:227-46.

[12] Kinloch AJ. Adhesion and adhesives - science and technology. London: Chapman and Hall; 1987.

[13] Rice JR. A path independent integral and the approximate analysis of strain concentration by notches and cracks. ASME J Appl Mech 1968;35:379-86.

[14] Paris AJ, Paris PC. Instantaneous evaluation of J and C. Int J Fract 1988;38:RI9-R21.

[15] Biel A, Stigh U. Strength and toughness in shear of constrained layers. Int J Solids Struct 2018;138:50-63.

[16] Olsson P, Stigh U. On the determination of the constitutive properties of thin interphase layers - an exact inverse solution. Int J Fract 1989;41:R71-6.

[17] Plante J-S, Dubowsky S. Large-scale failure modes of dielectric elastomer actuators. Int J Solids Struct 2006;43:7727-51.

[18] Zhao X, Suo Z. Electrostriction in elastic dielectrics undergoing large deformation. J Appl Phys 2008;104:123530.

[19] Mott PH, Dorgan JR, Roland CM. The bulk modulus and Poisson's ratio of "incompressible" materials. J Sound Vib 2008;312:572-5.

[20] Ashby MF, Jones DRH. Engineering materials 2: An introduction to microstructures, processing and design. Exeter: Pergamon Press; 1986.

[21] Townsend BW, Ohanehi DC, Dillard DA, Austin SR, Salmon F, Gagnon DR. Characterizing acrylic foam pressure sensitive adhesive tapes for structural glazing applications -Part I: DMA and ramp-to-fail results. Int J Adhes Adhes 2011;31:639-49.

[22] Townsend BW, Ohanehi DC, Dillard DA, Austin SR, Salmon F, Gagnon DR. Characterizing acrylic foam pressure sensitive adhesive tapes for structural glazing applications - Part II: Creep rupture results. Int J Adhes Adhes 2011;31:650-9.

[23] Carlberger T, Biel A, Stigh U. Influence of temperature and strain rate on cohesive properties of a structural epoxy adhesive. Int J Fract 2009;155:155-66.

[24] Stigh U. Mechanics of the double cantilever beam specimen. Int J Fract 1989;41:R15-7.

[25] lord Rayleigh (John William Strutt). Investigation of the character of the equilibrium of an incompressible heavy fluid of variable density. Proc London Mathe Soc 1883;14:170-7.

[26] Taylor GF. The instability of liquid surfaces when accelerated in a direction perpendicular to their planes. Proc Roy Soc London. Series A, Mathe Phys Sci 1950;201:192-6.

[27] Villey R, Creton C, Cortet PP, Dalbe MJ, Jet T, Saintyves B, et al. Rate-dependent elastic hysteresis during the peeling of pressure sensitive adhesives. Soft Matter 2015;11:3480-91. 